\title{
Alemtuzumab induction is safe for renal transplant recipients
}

Alemtuzumab is as safe and effective as commonly used induction agents in preventing biopsy-confirmed acute rejection, reports a study published in the New England Journal of Medicine.

Antibody induction therapy enables early glucocorticoid withdrawal in renal transplant recipients. The most frequently used induction agents in the US have been rabbit antithymocyte globulin and basiliximab. Alemtuzumab is a newer agent developed in the UK. "Early results with alemtuzumab in the US were highly variable and created a lot of concerns about infectious complications, rejection, and how well patients did," says investigator Michael Hanaway on behalf of the INTAC Study Group, who carried out the latest study following excellent results with the use of alemtuzumab from a 2005 study.

Renal transplant recipients from 30 centers in the USA were stratified according to immunological risk and randomly assigned to receive alemtuzumab or conventional induction therapy (basiliximab in patients at low immunological risk and rabbit antithymocyte globulin in patients at high risk of graft rejection). All patients received tacrolimus and mycophenolate mofetil as maintenance immunosuppression, and prednisolone was tapered and discontinued by day 5 after transplantation.

\section{4 ...the rate of biopsy-confirmed acute rejection was significantly lower among patients receiving alemtuzumab... 77}

The primary end point was the rate of biopsy-confirmed acute rejection at 6 months and 12 months. Secondary end points included efficacy, patient and graft survival, and infection. Patients were followed for 3 years to assess long-term safety and efficacy.

Overall, the rate of biopsy-confirmed acute rejection was significantly lower among patients receiving alemtuzumab than among those receiving conventional therapy, at both 6 months (3\% versus $15 \%$ ) and 12 months (5\% versus 17\%). Subgroup analyses at 3 years showed that acute rejection rates were lower with alemtuzumab than with conventional therapy in the low-risk group, but among high-risk patients rates of biopsyconfirmed acute rejection were similar in the alemtuzumab and rabbit antithymocyte globulin groups. Late acute rejection (occurring between months 12 and 36 after transplantation) was more common in the alemtuzumab group than in the conventional therapy group ( $8 \%$ versus $3 \%$ ), which prompted an accompanying editorial to note that use of alemtuzumab will require increased vigilance beyond the first year after transplantation.

Patient and graft survival were similar in conventional and alemtuzumab groups and the rates of infectious adverse events did not differ significantly.

"The questions not addressed in this study are how alemtuzumab compares with the other agents in terms of formation of antibodies against the donor kidney after transplantation," says Hanaway. "Future research in this area needs to make antibody formation and rejection mediated by these antibodies a focal point."

Helene Myrvang
Original article Hanaway, M. J. et al. Alemtuzumab induction in renal transplantation. N. Engl. J. Med. 364, 1909-1919 (2011) 